\title{
The influence of motion coherence manipulations on the synchronization level of a perception-action task
}

\author{
Tanja Ceux ${ }^{\mathrm{a}, *}$, Johan Wagemans ${ }^{\mathrm{b}}$, Pedro Rosas ${ }^{\mathrm{b}}$, Gilles Montagne ${ }^{\mathrm{c}}$, Martinus Buekers ${ }^{\mathrm{a}}$ \\ a Motor Learning Laboratory, Department of Kinesiology, Faculty of Kinesiology and Rehabilitation Sciences, \\ Katholieke Universiteit Leuven, Tervuursevest 101, 3001 Leuven, Belgium \\ ${ }^{\mathrm{b}}$ Laboratory for Experimental Psychology, Department of Psychology, Faculty of Psychology and Educational Sciences, \\ Katholieke Universiteit Leuven, Tiensestraat 102, 3000 Leuven, Belgium \\ c UMR “Mouvement et Perception”, Faculté des Sciences du Sport, Université de la Méditerranée et CNRS, \\ 163, avenue de Luminy, 13009 Marseille, France
}

Received 3 March 2005; received in revised form 3 March 2005; accepted 4 March 2005

Available online 12 April 2005

\begin{abstract}
The present experiment was conducted to examine the integration of the motion coherence paradigm in a synchronization task. Random-dot kinematograms were used to generate a pattern of oscillating dots representing four different coherence levels $(10 \%, 30 \%, 50 \%$ and $100 \%)$ and one target-alone condition. The participants had to synchronize their arm with the coherently moving dots according to two different synchronization modes (in-phase and anti-phase). The results revealed a substantial performance decline when the target/noise ratio dropped under the critical threshold situated around the $30 \%$ coherence level, albeit independent of the synchronization mode. In general, these findings highlighted the impact of the perception of motion based on the level of motion coherence in the visual signal on the synchronization behavior in a perception-action setting.
\end{abstract}

(C) 2005 Elsevier B.V. All rights reserved.

Keywords: Synchronization; Coherent motion; Random-dot kinematogram; Phase manipulations; Perception-action coupling

\section{Introduction}

The precision of the interplay between an actor and the environment constitutes a vital element in achieving successful human behavior. The accuracy level of this perception-action interaction is quite remarkable. Not only because of the constantly changing surroundings, but also due to the proper movements of the actor, which add considerably to the dynamic nature of the perception-action cycle. Image motion processing plays an important role in the smooth operation of this cycle and delimits to some degree the appropriateness of the actions in a given environment. Thus, such activities as driving a car, crossing the road, catching a ball, and synchronizing movements of body segments to visual stimuli

\footnotetext{
* Corresponding author. Tel.: +32 16329069; fax: +32 16329197.

E-mail address: tanja.ceux@faber.kuleuven.be (T. Ceux).
}

strongly rely on the ability to accurately perceive, interpret and predict the motion of the constituting elements of the surroundings.

At present, the literature provides a good understanding of the lower motion areas in which directionally selective cells, located in the primary visual cortex (area VI), detect motion in each possible direction at each distinct position of the image [6,14]. It should not be very surprising then that research currently focuses on explaining the higher motion areas requiring the organization and integration of simple local motions initiated by the middle temporal area (MT/V5). An important (dynamic) factor in this perceptual image organization is the well-known Gestalt notion of "common fate". This perceptual grouping principle states that elements moving coherently in the same direction and at the same speed have a strong tendency to be grouped together $[11,16]$. 
One of the best means to investigate the mechanisms that determine which signal units of a complex scene belong together and which units do not, relates to the so-called motion coherence paradigm. In these studies random-dot kinematograms are used in which rapid position changes of many randomly positioned elements provokes the perception of elements in motion [11]. These random-dot kinematograms are characterized by some fraction of dots (signal dots) moving coherently in the same direction, while the remaining dots (noise) are moving in a random or incoherent way. Since each dot in the kinematogram can be controlled independently, it is possible to manipulate the coherence levels of the display by adding randomly moving dots to observe how well the participants perform a discrimination task under different noise levels. Performance in a motion perception task with such a stimulus (e.g. discriminating between two opposite motion directions) is then related to the so-called coherence threshold, expressed as the percentage of dots moving in the same direction at the same speed [7,9,10,13,16-18]. Depending on the speed and on the duration of the coherent motion, and on the area and visual field of the visual stimulus, the coherence thresholds for human and non-human primate observers were found to be within the range of 2 and $30 \%$ [2,5]. In essence, the consistent psychometric relationship between the strength of the coherent motion signal and the ability to discriminate the motion direction in the random-dot kinematogram can be regarded as a further refinement of the perceptual grouping principle of 'common fate'.

In the present study we want to take this perceptual issue a step further and apply it to the action domain. More specifically, we will make use of the motion coherence paradigm to investigate how coherence manipulations influence the synchronization performance. In fact, in a preliminary experiment we tested 18 participants to validate the use of the different motion coherence levels in a perception-action setting. This pilot study showed the successful integration of the motion coherence paradigm as the synchronization behavior varied considerably between a limited number of motion coherence levels in the range of $10 \%$ and $100 \%$. More specifically, lower coherence levels, i.e. a decreased target/noise ratio, provoked poorer synchronization performance when the participants had to synchronize their arm movement to the coherently moving target surface. Using this information, two major questions were addressed in the present experiment. Firstly, we manipulated the spatio-temporal characteristics of the visual signal to further elaborate the impact of different coherence levels on the synchronization action. The degree to which the coherence manipulation will influence synchronization performance will be highly informative to get a better idea about the strength of the perception-action coupling for this type of tasks. Secondly, this latter assumption will be examined more closely by introducing a phase manipulation. Buekers et al. [3] showed that synchronization performance was best when arm movements were executed in the same direction as the horizontal moving signal dot (in-phase). Conversely, synchronization behavior was poorer when par- ticipants had to perform the anti-phase synchronization mode involving an opposite movement direction as the target signal. Actually, by comparing the effect of the coherence manipulations on both in-phase and anti-phase movements, we can test whether the coupling strength is substantially stronger when perception and action spatially match. Finally, it was anticipated that (1) given the findings of the pilot experiment a performance gain can be predicted when the motion coherence, i.e. the target/noise dots ratio, increases, and (2) synchronization performance is expected to be better in the case of in-phase synchronization (with stronger perception-action coupling than in the anti-phase condition). Hence, we stated performance to be best in the in-phase condition for the highest coherence level and worst in the anti-phase for the lowest coherence level. How the two factors motion coherence and synchronization mode will interact in the intermediate coherence conditions is more difficult to predict.

\section{Materials and methods}

\subsection{Participants}

Fifteen female students (mean age $=19.72$ years, age range 18-23) volunteered to participate in the study. The participants had a normal or corrected to normal vision and had no previous experience with the task, nor were they aware of the goal of the study. Prior to testing, all the participants gave their informed consent.

\subsection{Apparatus}

Participants were seated comfortably on a chair behind a table in a darkened room. The right forearm had to be placed on a lever that could freely rotate in the horizontal plane. While the participant's elbow was positioned just above the lever's axis of rotation, he/she had to grab a handle that was fixed to the distal end of the lever. A shaft encoder (Tamagawa TS5116) was connected to the axis of the lever and recorded its position (accuracy of $0.1^{\circ}$ ) at a rate of $100 \mathrm{~Hz}$. The visual signal was projected on a white wall in front of the participants at a viewing distance of $145 \mathrm{~cm}$ by means of an LCD-projector with a spatial resolution of $800 \times 600$ pixels, and materialized as a black rectangle $(117 \mathrm{~cm} \times 119 \mathrm{~cm})$ filled with the total number of 1000 dots. The maximum luminance in the stimuli was approximately $120 \mathrm{~cd} / \mathrm{m}^{2}$ (corresponding to the dots), while the background luminance was $15 \mathrm{~cd} / \mathrm{m}^{2}$. The dynamic display was a random-dot kinematogram, created in MATLAB, using the Psychophysics Toolbox extensions [1,12]. It consisted of a fraction of target dots moving coherently back and forth from left to right, while the remaining dots (noise) were moving in a random or incoherent way. All dots were moving at a sinusoidal mean velocity of $26.06 \%$ s. Between the two reversal positions of the display, all dots had a lifetime of five frames $(0.080 \mathrm{~s})$ of the total signal sequence of 100 frames $(1.65 \mathrm{~s})$.

\subsection{Task and procedure}

The participants completed two synchronization modes (inphase versus anti-phase) in five experimental signal conditions representing four different coherence levels $(100 \%, 50 \%, 30 \%$ and 
$10 \%)$ and one target-alone condition. Since in this latter condition only one target dot was shown it could serve as a reference condition to define the mere impact of the coherent motion on the synchronization performance. In the $100 \%$ coherence condition all dots served as target dots and moved coherently back and forth from left to right. However, in the $10 \%$ coherence condition only $10 \%$ of the dots moved coherently. In this latter condition, $90 \%$ of the dots moved in a random way (noise). The $30 \%$ and the $50 \%$ coherence conditions were composed in a comparable manner. While the participants were instructed to synchronize the arm movement to the coherently moving signal dots in the same direction in the in-phase synchronization mode, the reversed coupling between arm movement and visual signal was required in the anti-phase mode.

For the target-alone condition participants had to move in the same direction as the one target dot in the in-phase mode while arm movements had to be performed in the opposite direction in the anti-phase mode. Each synchronization mode consisted of 60 reversal movements, divided in three trials of 20 cycles each. Every movement cycle lasted $3.3 \mathrm{~s}$ resulting in a total trial duration of $66 \mathrm{~s}$ for each condition.

The sequence of the 10 conditions (signal condition $x$ synchronization mode) was randomized between the participants and the arm movement had to be initiated when a single target dot completed 1 (in-phase) or 1.5 (anti-phase) back and forth cycle. The required amplitude for the arm movement was imposed by the target dots and was equal to $43^{\circ}$.

\subsection{Data analysis}

The mean movement amplitude $\left(^{\circ}\right)$ and mean within-participant variability of the amplitude $\left(^{\circ}\right)$ were calculated for each participant to examine the spatial accuracy of the arm movement according to the criterion amplitude of the visual signal $\left(43^{\circ}\right)$. In addition to these spatial parameters, the variable error $(\mathrm{ms})$ was used to indicate the temporal variability at both the left and right reversal position. Finally, the mean absolute error of the (continuous) relative phase $\left.{ }^{\circ}\right)$ and the mean within-participant variability of the (continuous) relative phase $\left(^{\circ}\right.$ ) were computed for each participant to study the spatio-temporal characteristics of the synchronization performance at each point in the trajectory. Specifically, the absolute error of the (continuous) relative phase, indicating the absolute difference in phase angle between the arm movement and the visual signal, was used as a measure of performance accuracy while its standard deviation expressed the consistency of the synchronization behavior. All these dependent variables were computed as the average of the three trials in each synchronization mode of the five different signal conditions for each participant.

Statistical analyses consisted of a $5 \times 2$ (signal condition $\times$ synchronization mode) factorial design with repeated measures on both factors. The alpha-level was set at $p<0.05$, and all significant results involving more than two means were analyzed using Tukey's HSD procedures.

\section{Results}

\subsection{Spatial parameters}

Statistical analysis of the amplitude showed a significant main effect for the experimental signal condition $(F(4$,
14) $=5.106, p=0.0014$ ) (see Fig. 1). In fact, arm movements became significantly larger and less accurate in the targetalone condition, where only one target dot was presented, than in the $10 \%$ and the $30 \%$ coherence conditions, where respectively 90 and $70 \%$ of the total number of dots was noise moving in a random way (see Table 1). Furthermore, the results for the movement amplitude varied significantly as a function of the synchronization mode $(F(1,56)=4.960$, $p=0.0429$ ). Actually, the best spatial precision was achieved in the in-phase synchronization mode where arm movements had to be executed in the same direction as the coherently moving target dots. In other words, the obtained amplitude deviated significantly more from the criterion target amplitude of $43^{\circ}$ in the anti-phase condition.

Contrary to these results, the within-participant variability of the movement amplitude increased as the number of coherently moving dots decreased $(F(4,14)=23.052$, $p<0.0001$ ), albeit independent of the synchronization mode. Specifically, both the $10 \%$ and the $30 \%$ coherence conditions revealed substantial performance deterioration compared to the three remaining experimental signal conditions $(50 \%$, $100 \%$ and target-alone). What's more, the consistency of the arm movement in the $50 \%$ coherence condition also meaningful differed from the performance in the $100 \%$ and target-alone conditions.

\subsection{Temporal parameters}

The analysis of the variable error, expressing the temporal variability of the arm movements only at both reversal positions, revealed a significant main effect for the signal condition $((F(4,14)=10.028, p<0.0001)$ at the left side and $F(4$, $14)=10.440, p<0.0001$ for the right side). Tukey/Kramer post-hoc analyses demonstrated a significant performance decline at the left and right reversal point in the $10 \%$ coherence condition (containing the largest number of noise dots) as compared to the four other signal conditions $(30 \%, 50 \%$, $100 \%$ and target-alone). As can be seen in Table 1, extremely high variability scores were observed in the $10 \%$ coherence condition which illustrated the participants' inability to extract the coherent moving target dots in this lowest coherence level. Note, however, that the $100 \%$ and the target-alone conditions produced fairly normal variable errors. Thus, when coherence levels were high, synchronization performance was good. Based on these data, it can be concluded that increasing the number of randomly moving noise dots elicited serious problems to reverse the arm in a consistent way.

Additionally, both the results of the variable error at the left as well as the right reversal position failed to show any differences in temporal consistency of the synchronization behavior between the in-phase and anti-phase coordination mode.

\subsection{Spatio-temporal parameters}

The ANOVA on the absolute error of (continuous) relative phase indicated a significant difference between the five 


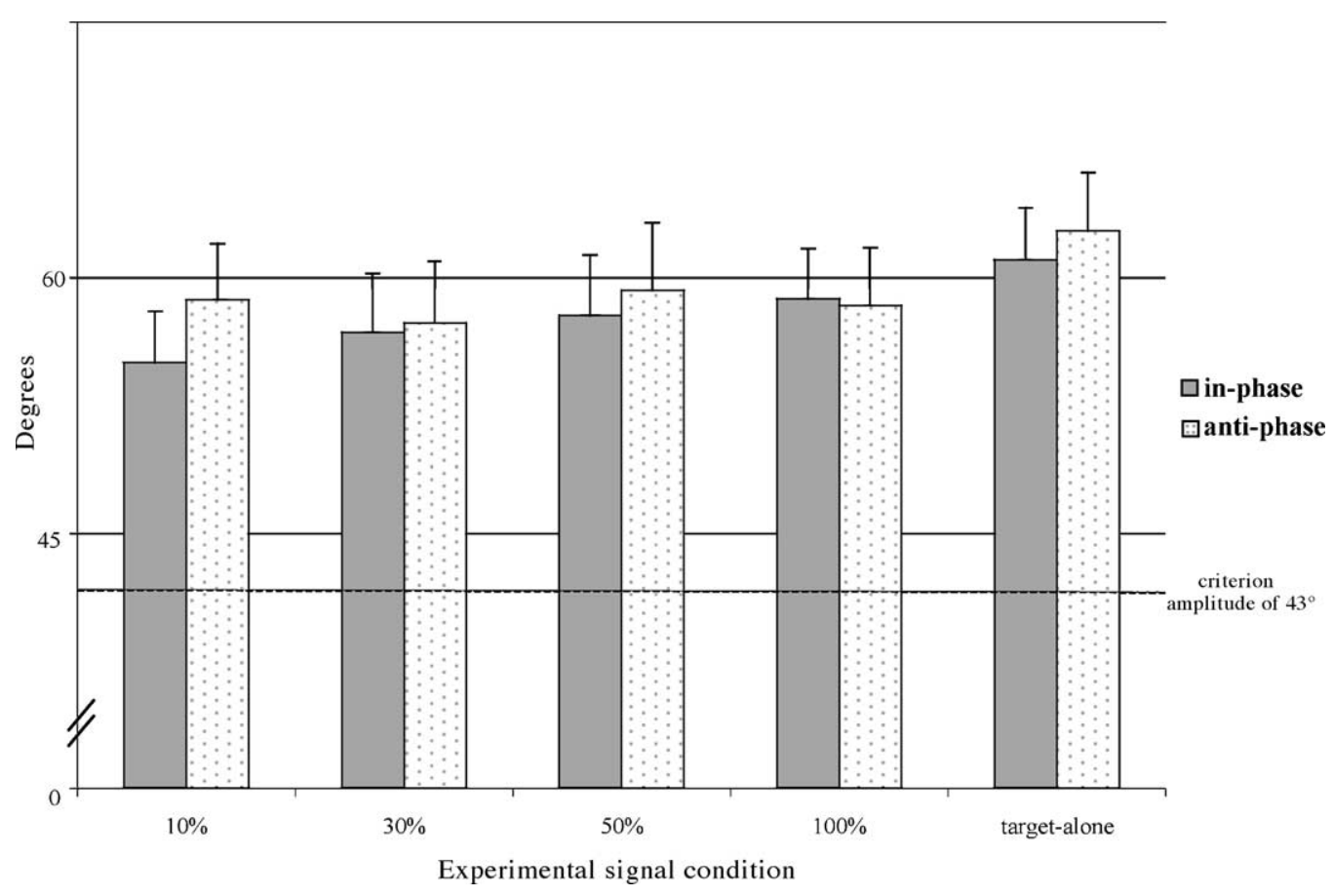

Fig. 1. Mean and standard error of the movement amplitude.

signal conditions $(F(4,14)=4.845, p=0.0020)$. Post-hoc analyses revealed that the spatio-temporal characteristics of the arm movement in the 10 and 30\% coherence conditions differed from the target-alone condition. What's more, meaningful performance difference was also found in the lowest coherence level, i.e. the $10 \%$ coherence condition, as compared to the highest coherence level, i.e. the $100 \%$ coherence condition. As can be seen in Fig. 2, an even more pronounced performance decrease was observed for the within-participant variability of (continuous) relative phase
$(F(4,14)=17.263, p<0.0001)$. More specifically, the $10 \%$ coherence condition differed significantly from all other signal conditions and also the synchronization performance in the $30 \%$ coherence condition was more variable than in the target-alone condition.

In addition, a significant interaction effect between signal condition and synchronization mode was obtained $(F(4$, $14)=2.749, p=0.0370)$. Performing arm movements in the same direction as the target dots, i.e. the in-phase synchronization mode, resulted in better synchronization behavior as

Table 1

Mean of the dependent synchronization measurements

\begin{tabular}{|c|c|c|c|c|c|}
\hline & $10 \%$ & $30 \%$ & $50 \%$ & $100 \%$ & Target-alone \\
\hline \multicolumn{6}{|l|}{ Amplitude $\left(^{\circ}\right)$} \\
\hline In-phase & 55.0 & 56.8 & 57.8 & 58.7 & 61.0 \\
\hline Anti-phase & 58.7 & 57.3 & 59.2 & 58.4 & 62.7 \\
\hline \multicolumn{6}{|c|}{ Within-participant variability of amplitude $\left(^{\circ}\right)$} \\
\hline In-phase & 10.6 & 9.2 & 7.1 & 5.3 & 5.4 \\
\hline Anti-phase & 10.3 & 7.8 & 7.8 & 5.9 & 4.7 \\
\hline \multicolumn{6}{|c|}{ Variable error left (ms) } \\
\hline In-phase & 1064 & 379 & 151 & 125 & 75 \\
\hline Anti-phase & 776 & 255 & 261 & 144 & 105 \\
\hline \multicolumn{6}{|c|}{ Variable error right (ms) } \\
\hline In-phase & 1111 & 390 & 145 & 127 & 80 \\
\hline Anti-phase & 749 & 247 & 227 & 135 & 105 \\
\hline \multicolumn{6}{|c|}{ Absolute error of (continuous) relative phase $\left(^{\circ}\right.$ ) } \\
\hline In-phase & 36 & 22 & 17 & 17 & 13 \\
\hline Anti-phase & 28 & 31 & 27 & 19 & 13 \\
\hline \multicolumn{6}{|c|}{ Within-participant variability of (continuous) relative phase $\left({ }^{\circ}\right)$} \\
\hline In-phase & 35 & 20 & 15 & 15 & 11 \\
\hline Anti-phase & 28 & 21 & 20 & 16 & 13 \\
\hline
\end{tabular}




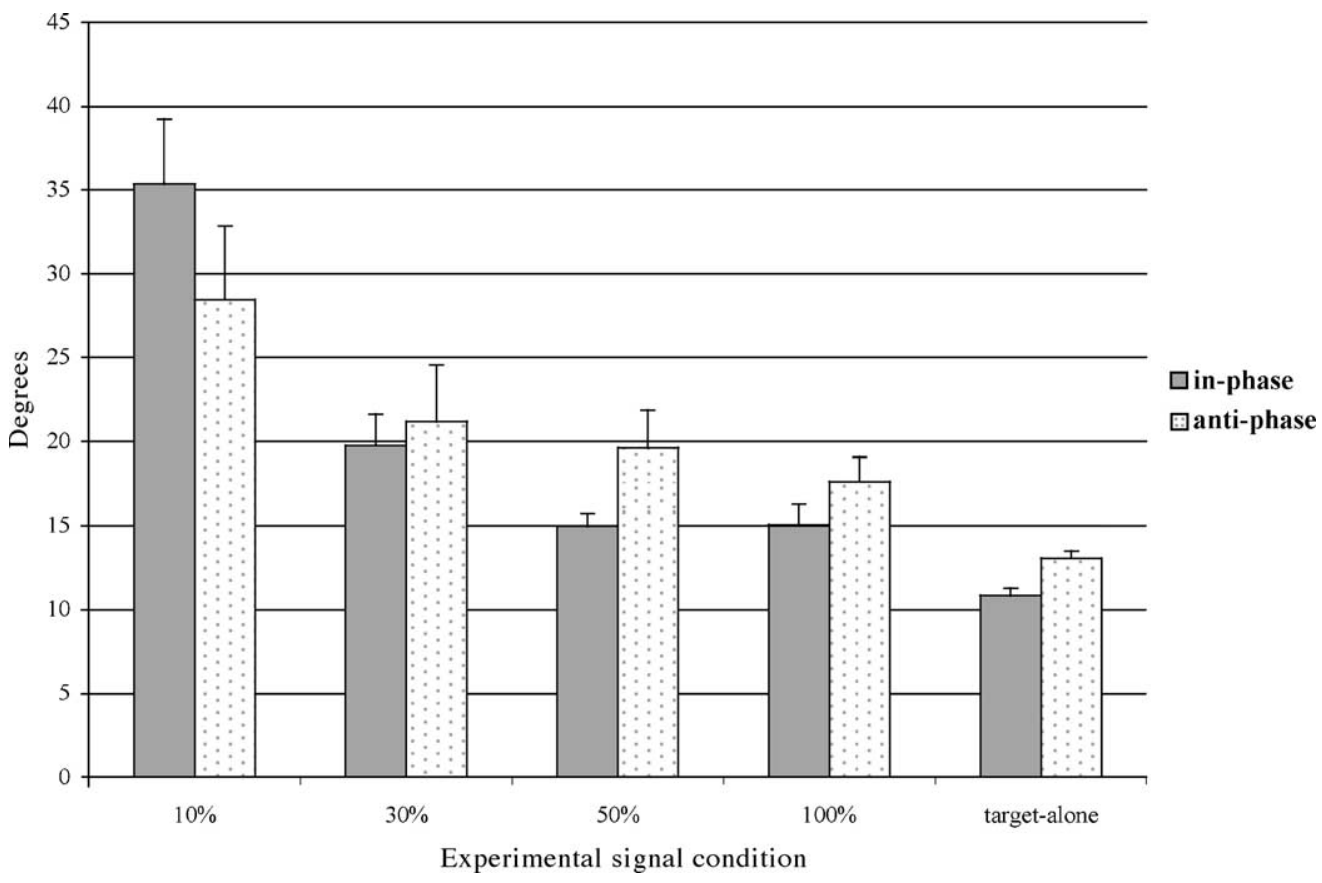

Fig. 2. Mean and standard error of the within-participant variability of relative phase.

compared to the anti-phase synchronization mode in which the participants had to move in the opposite direction with the exception of the $10 \%$ coherence condition. Actually, when the motion coherence level enables a more or less synchronized behavior, performance is significantly better in the in-phase synchronization mode as compared to the anti-phase mode.

\section{Discussion}

Despite the widely accepted use of the perception of motion based on the level of motion coherence in the visual signal as a performance measure in visual motion processing, little or nothing is known about its operation within the framework of actions. The findings of our recent studies on synchronization [3,4], challenged us to apply the coherence paradigm to this type of task. Therefore, the present experiment was set up to determine more thoroughly the role of the motion coherence paradigm on the synchronization performance. For this reason, and in addition to the coherence manipulations, a phase manipulation was introduced.

To do so, the visual signal consisted of random-dot kinematograms with different coherence levels in which the coherently moving surface of signal dots had to be extracted and tracked by the participants with their right arm. Based on the findings of the above mentioned pilot experiment, four signal conditions representing different coherence levels, namely a $10 \%, 30 \%, 50 \%$ and $100 \%$ target/noise dots ratio were used. In addition to these complex displays, a target-alone condition was incorporated in the present study, serving as an overall reference condition.
As the results of the pilot experiment indicated, there were no problems implementing the motion perception coupled to the level of motion coherence in the context of a synchronization task. Given this observation, we could focus on the question as to how different coherence levels would influence synchronization performance. As mentioned above, we anticipated that degrading perception by decreasing the number of target dots in proportion to the number of noise dots would deteriorate synchronization performance. As expected, the data demonstrated a disturbance of synchronization behavior due to a decreasing target/noise ratio. Particularly, the spatial consistency of the movement already showed performance decline when the $50 \%$ coherence level was achieved. Further, the results of the temporal and spatio-temporal parameters (absolute relative phase and within-participant variability of relative phase) revealed synchronization problems mainly situated at the $10 \%$ coherence level. These findings clearly illustrate that synchronization performance was seriously affected when the number of randomly moving noise dots reached a critical level. As such this observation is in line with perceptual observation studies [7,9,10,13,16-18], expressing performance problems in terms of particular coherence thresholds (in percentage). Apparently, for the spatial variability this threshold seemed to be situated around the 50\% coherence condition. For the variable error (left and right) and the (continuous) relative phase, the threshold was located between the $10 \%$ and the $30 \%$ coherence levels. Note that the present experiment intended to investigate the role of the motion coherence paradigm in a synchronization task rather than determining the perceptual coherence threshold for accurate synchronization performance as such. 
The second point of interest was investigated by adding a phase manipulation to the motion coherence manipulation. At first sight, the majority of the dependent measures failed to confirm the results of previous research $[3,4,15,20]$ since the overall synchronization performance was similar for the in-phase and anti-phase mode. Only the results of the movement amplitude showed less accurate arm movements in all signal condition when the task was completed according to the anti-phase mode. Most probably the motion coherence manipulation was responsible for this unexpected result. For instance, when synchronization problems materialized in the $10 \%$ signal condition, the advantage of the in-phase condition disappeared, leading to similar performance levels for both synchronization modes. Vice versa, when participants had no problems detecting the coherently moving target signal, i.e. in the $50 \%, 100 \%$ and target-alone conditions, performance became less accurate and more variable in the antiphase condition than in the in-phase synchronization mode. As Fig. 2 illustrated, this explanation was confirmed by the signal condition $\times$ synchronization mode interaction effect on the within-participant variability of relative phase. Based on these findings, we can conclude that the critical and dominant impact of the motion coherence manipulation foiled a possible positive phase effect for the in-phase synchronization mode. Actually, the lack of general statistical difference between the in-phase and anti-phase mode was also found when finger movements were established by moving in-phase or synchronization (i.e. movement coincided with each tone) and anti-phase or syncopation (i.e. movement occurred directly in between consecutive tones) with an auditory metronome [8]. In particular, the behavioral data (relative phase and standard deviation of the relative phase) showed no difference between the conditions as movements were timed consistently for the synchronization and syncopation modes. However, a broader neural network of activation was engaged during syncopation (anti-phase) compared with synchronization (in-phase), including increased activation in supplementary motor area, left premotor area, right thalamus, bilateral inferior gyrus and cerebellum. These results illustrate that the cortical and subcortical areas recruited to support a simple motor timing task depend crucially on the method used to establish the temporal reference. Thus, it might be interesting for future research to investigate the neural correlates of the present synchronization task.

An additional finding of the present study that is noteworthy relates to the observed amplitudes for the target-alone condition. Actually, the movement amplitudes were surprisingly larger in this latter condition as compared to the $10 \%$ and $30 \%$ coherence levels. It is tempting to associate this observation to the work of Xia and Barnes [19], as it revealed the importance of a 'certain' degree of target complexity and irregularity to ameliorate performance in an oculo-manual tracking task. In fact, the coupling between hand and eye motion became tighter when the complexity and irregularity of the stimulus increased. In our study, the participants that were executing the target-alone condition did not ex- perience the same target complexity as the one used in the coherence conditions. Possibly this discrepancy provoked the amplitude effects observed in the present experiment. Nevertheless, the results of this study support the suitability of random-dot kinematograms (size of the display, total number of dots, lifetime, velocity, luminance, ...) to investigate the influence of motion coherence in synchronization tasks.

To summarize, the results clearly demonstrated the influence of motion coherence paradigm on the quality of the synchronization performance in a perception-action setting. Actually, synchronization performance was seriously affected when the perceptual conditions were complex, i.e. as the number of noise dots dropped under the $30 \%$ coherence level. However, this latter effect was independent of phase manipulations, i.e. the in-phase or anti-phase synchronization mode. Thus, when both coherence and phase manipulations are applied to synchronization behavior, the coherence levels will exert their dominant role.

\section{Acknowledgement}

We thank Frans A.J. Verstraten for providing us the initial Motion Coherence MATLAB code.

\section{References}

[1] Brainard DH. The Psychophysics Toolbox. Spat Vis 1997;10:433-6.

[2] Britten KH, Shadlen MN, Newsome WT, Movshon JA. The analysis of visual motion: a comparison of neuronal and psychophysical performance. J Neurosci 1992;12:4745-65.

[3] Buekers MJ, Bogaerts HP, Swinnen SP, Helsen WF. The synchronization of human arm movements to external events. Neurosci Lett 2000;290:181-4.

[4] Ceux T, Buekers MJ, Montagne G. The effects of enhanced visual feedback on human synchronization. Neurosci Lett 2003;349:103-6.

[5] Croner LJ, Albright TD. Image segmentation enhances discrimination of motion in visual noise. Vision Res 1997;37:1415-27.

[6] Gegenfurtner K. Visual psychophysics: synchrony in motion. Nat Neurosci 1998;1:96-8.

[7] Grzywacz NM, Watamaniuk SN, McKee SP. Temporal coherence theory for the detection and measurement of visual motion. Vision Res 1995;35:3183-203.

[8] Jantzen KJ, Steinberg FL, Kelso JA. Brain networks underlying human timing behavior are influenced by prior context. Proc Natl Acad Sci USA 2004;101:6815-20.

[9] Nakayama K. Biological image motion processing: a review. Vision Res 1985;25:625-60.

[10] Newsome WT, Pare EB. A selective impairment of motion perception following lesions of the middle temporal visual area (MT). J Neurosci 1988;8:2201-11.

[11] Palmer SE. Perceiving motion and events. In: Vision science: photons to phenomenology. Cambridge, MA: MIT Press; 1999. p. 466504.

[12] Pelli DG. The Video Toolbox software for visual psychophysics: transforming numbers into movies. Spat Vis 1997;10:437-42.

[13] Scase MO, Braddick OJ, Raymond JE. What is noise for the motion system? Vision Res 1996;36:2579-86.

[14] Snowden RJ, Verstraten FA. Motion transparency: making models of motion perception transparent. Trends Cogn Sci 1999;3:369-77. 
[15] Swinnen SP, Jardin K, Meulenbroek R, Dounskaia N, HofkensVan Den Brandt M. Egocentric and allocentric constraints in the expression of patterns of interlimb coordination. J Cogn Neurosci 1997;9:348-77.

[16] Uttal WR, Spillmann L, Sturzel F, Sekuler AB. Motion and shape in common fate. Vision Res 2000;40:301-10.

[17] Watamaniuk SN, McKee SP, Grzywacz NM. Detecting a trajectory embedded in random-direction motion noise. Vision Res 1995; 35:65-77.
[18] Watamaniuk SN, Sekuler R. Temporal and spatial integration in dynamic random-dot stimuli. Vision Res 1992;32:23417.

[19] Xia R, Barnes G. Oculomanual coordination in tracking of pseudorandom target motion stimuli. J Mot Behav 1999;31:2138

[20] Zaal FT, Bingham GP, Schmidt RC. Visual perception of mean relative phase and phase variability. J Exp Psychol Hum Percept Perform 2000;26:1209-20. 